\title{
Recurrent or refractory trigeminal neuralgia after microvascular decompression, radiofrequency ablation, or radiosurgery
}

\author{
Rene O. Sanchez-Mejia, M.D., Mary Limbo, B.A., Jason S. Cheng, B.S., \\ Joaquin Camara, B.S., Mariann M. Ward, R.N., M.S., and Nicholas M. Barbaro, M.D. \\ Department of Neurological Surgery, University of California, San Francisco, California
}

\begin{abstract}
Object. Trigeminal neuralgia (TN) is characterized by paroxysmal lancinating pain in the trigeminal nerve distribution. When TN is refractory to medical management, patients are referred for microvascular decompression (MVD), radiofrequency ablation, or radiosurgery. After the initial treatment, patients may have refractory or recurrent symptoms requiring retreatment. The purpose of this study was to determine what factors are associated with the need for retreatment and which modality is most effective.

Methods. To define this population further, the authors evaluated a cohort of patients who required retreatment for $\mathrm{TN}$. The mean follow-up periods were 51 months from the first treatment and 23 months from the last one, and these were comparable among treatment groups.

Conclusions. Trigeminal neuralgia can recur after neurosurgical treatment. In this study the authors demonstrate that the number of patients requiring retreatment is not negligible. Lower retreatment rates were seen in patients who initially underwent radiosurgery, compared with those in whom MVD or radiofrequency ablation were performed. Radiosurgery was more likely to be the final treatment for recurrent TN regardless of the initial treatment. After retreatment, the majority of patients attained complete or very good pain relief. Pain relief after retreatment correlates with postoperative facial numbness.
\end{abstract}

\section{KEY WORDS • trigeminal neuralgia • microvascular decompression • rhizotomy • radiosurgery - recurrence}

The clinical syndrome of TN or tic douloureux,,$^{5}$ which affects 0.004 to $0.016 \%$ of the US population, is characterized by paroxysmal lancinating pain in the distribution of the trigeminal nerve. ${ }^{5,11,12}$ Pain, which is usually unilateral and more common in the $\mathrm{V}_{2}$ and $\mathrm{V}_{3}$ roots, can be triggered by a variety of trigeminal sensory inputs. The origins of TN are not entirely understood, but in many cases the cause is trigeminal nerve root compression at the brainstem due to a vascular structure or mass lesion. ${ }^{12}$ When medical management-typically with anticonvulsant drugs such as carbamazepine or gabapentin-is not successful, patients are referred for neurosurgical treatment.

The treatment of patients with medically refractory $\mathrm{TN}$ has evolved to include multiple modalities, such as MVD, radiofrequency ablation, and radiosurgery. ${ }^{11}$ Microvascular decompression was the first technique to be proven efficacious for the treatment of $\mathrm{TN}$, and it results in excellent or good outcomes in approximately $80 \%$ of patients. ${ }^{2,8}$ Never-

Abbreviations used in this paper: MVD = microvascular decompression; $\mathrm{TN}=$ trigeminal neuralgia; $\mathrm{V}_{1}=$ first division of the trigeminal nerve; $\mathrm{V}_{2}=$ second division of the trigeminal nerve; $\mathrm{V}_{3}=$ third division of the trigeminal nerve. theless, $5 \%$ of patients who undergo MVD experience little or no pain relief, and 10 to $20 \%$ suffer recurrent symptoms requiring retreatment. ${ }^{1,2,4}$ Recurrent symptoms typically occur after a mean of 1.9 pain-free years, with a yearly recurrence risk of 1 to $4 \%{ }^{2,4}$ In addition, refractory and recurrent symptoms can occur after radiosurgery or radiofrequency ablation. After the latter procedure, 21 to $50 \%$ of patients have recurrent TN and $15 \%$ require retreatment. ${ }^{13,15}$ After radiosurgery, approximately 13 to $22 \%$ of patients require repeated treatment for recurrent symptoms. ${ }^{3,7,16}$

Patients with persistent or recurrent symptoms after neurosurgical treatment may require retreatment if the symptoms are severe. In this paper we evaluate a cohort of patients who required retreatment. We have excluded patients with multiple sclerosis, given that the pathophysiological features of their TN are thought to differ from those of idiopathic TN. We demonstrate that there is a population of patients who require retreatment, that good outcomes can be obtained with retreatment, that initial treatment with radiosurgery may diminish the need for further procedures, and that postoperative control of TN correlates with trigeminal numbness. 


\section{CLINICAL MATERIAL AND METHODS}

\section{Patient Population}

Our database of 209 patients who underwent neurosurgical treatment for TN between 1997 and 2004 was analyzed retrospectively after permission was obtained from the University of California, San Francisco's Committee on Human Research. We started our study with patients treated in 1997 because that was the 1st year in which computerized records were kept at the University of California, San Francisco. Between 1997 and 2004, 32 (15.3\%) of 209 patients with TN (19 women and 13 men) required retreatment at our institution (see Table 1 for patient characteristics). As shown in Table 2, the mean age of the retreated patients was $69.1 \pm 12.3$ years (range 51-91 years), and the mean age at symptom onset was $53.7 \pm 15.5$ years (range 30-81 years). Patients who had undergone prior treatment at other institutions were excluded because we were unable to obtain the information needed for analysis and because the exact method of treatment was hard to ascertain. Patients with multiple sclerosis were also excluded and analyzed separately, as mentioned earlier.

Of the patients eligible for analysis, 32 with refractory or recurrent symptoms required retreatment. One neurosur- geon (N.B.) performed the additional treatments in all patients. Radiosurgery was performed with a gamma knife apparatus in conjunction with the Department of Radiation Oncology.

Patients were evaluated for demographic information, clinical presentation including symptoms and distribution of facial pain, initial treatment modality, comorbidities, time elapsed to recurrence and severity of symptoms, retreatment modalities, and final outcomes with respect to pain control and paresthesias. Preoperative and postoperative medications and doses were recorded, and any surgical complications were noted. If vascular compression of the trigeminal nerve root was present at the time of surgery, the specific vessel compressing the root was recorded. Outcomes were assessed by determining the extent of pain relief, the frequency of ongoing painful episodes, and the requirements for continuing medication. The Student t-test was used for comparisons between two groups and a probability value less than 0.05 was considered significant.

\section{RESULTS}

The mean duration of preoperative symptoms was simi-

TABLE 1

Characteristics of 32 patients who required retreatment for refractory or recurrent $T N^{*}$

\begin{tabular}{|c|c|c|c|c|c|c|c|c|c|}
\hline \multirow{2}{*}{$\begin{array}{l}\text { Case } \\
\text { No. }\end{array}$} & \multirow{2}{*}{$\begin{array}{l}\text { Age at Tx } \\
(\mathrm{yrs}), \text { Sex }\end{array}$} & \multirow{2}{*}{$\begin{array}{l}\text { Age at } \mathrm{Sx} \\
\text { Onset (yrs) }\end{array}$} & \multirow{2}{*}{$\begin{array}{c}\text { Preop Sx } \\
\text { Duration (mos) }\end{array}$} & \multirow[b]{2}{*}{ Side } & \multirow{2}{*}{$\begin{array}{l}\text { Nerve } \\
\text { Division }\end{array}$} & \multirow[b]{2}{*}{ Tx Plan } & \multicolumn{2}{|c|}{ FU (mos) } & \multirow[b]{2}{*}{ Pain Relief $(\%)$} \\
\hline & & & & & & & Last Tx & 1 st Tx & \\
\hline 1 & $56, \mathrm{~F}$ & 46 & 96 & lt & $\mathrm{V}_{2}$ & 1. MVD + rhiz; 2. RS; 3. RS & 24 & 41 & 100 \\
\hline 2 & $66, \mathrm{~F}$ & 56 & 134 & $\mathrm{rt}$ & $\mathrm{V}_{3}^{2}$ & 1. RS; 2. MVD; $3 . \mathrm{RF}$ & 12 & 29 & 100 \\
\hline 3 & $61, \mathrm{M}$ & 57 & 54 & lt & $\mathrm{V}_{2}+\mathrm{V}_{3}$ & 1. MVD + rhiz; 2. RS; 3. RS & 36 & 49 & 50 \\
\hline 4 & $77, \mathrm{~F}$ & 60 & 123 & $\mathrm{rt}$ & $\mathrm{V}_{2}^{2}+\mathrm{V}_{3}^{3}$ & 1. RF; 2. RS & 72 & 132 & 75 \\
\hline 5 & $55, \mathrm{~F}$ & 43 & 145 & bilat & $\mathrm{V}_{2}^{2}+\mathrm{V}_{3}^{3}$ & $\begin{array}{l}\text { 1. RS (rt); 2. MVD (lt); 3. MVD } \\
(\mathrm{rt}) ; 4 \text {. RS (bilat); 5. RS (lt side) }\end{array}$ & 6 & 66 & 75 \\
\hline 6 & $69, \mathrm{~F}$ & 43 & 300 & $\mathrm{rt}$ & $\mathrm{V}_{3}$ & 1. MVD; 2. RF; 3. RS & 18 & 35 & 100 \\
\hline 7 & $83, \mathrm{~F}$ & 71 & 108 & lt & $\mathrm{V}_{2}^{3}+\mathrm{V}_{3}$ & 1. RF; 2. RS & 12 & 45 & 100 \\
\hline 8 & $85, \mathrm{M}$ & 81 & 36 & $\mathrm{rt}$ & $\mathrm{V}_{1}^{2}+\mathrm{V}_{3}^{3}$ & 1. RS; 2. MVD & 2 & 10 & 100 \\
\hline 9 & $82, \mathrm{~F}$ & 67 & 131 & lt & $\mathrm{V}_{2}+\mathrm{V}_{3}^{3}$ & 1. MVD + rhiz; 2. RS; 3. RS & 36 & 79 & 100 \\
\hline 10 & $69, \mathrm{~F}$ & 62 & 21 & $\mathrm{rt}$ & $\mathrm{V}_{2}^{2}+\mathrm{V}_{3}^{3}$ & 1. MVD + rhiz; $2 . \mathrm{RS}$ & 12 & 42 & 50 \\
\hline 11 & $60, \mathrm{M}$ & 46 & 81 & $\mathrm{rt}$ & $\mathrm{V}_{2}^{2}+\mathrm{V}_{3}^{3}$ & 1. MVD + rhiz; 2. RS & 18 & 52 & 50 \\
\hline 12 & $91, \mathrm{~F}$ & 80 & 132 & $\mathrm{rt}$ & $\mathrm{V}_{2}^{2}+\mathrm{V}_{3}^{3}$ & 1. RF; 2. RS & 6 & 12 & 100 \\
\hline 13 & $70, \mathrm{~F}$ & 58 & 120 & $\mathrm{rt}$ & $\mathrm{V}_{2}^{2}$ & 1. MVD + rhiz; 2. RS & 24 & 48 & 100 \\
\hline 14 & $58, \mathrm{~F}$ & 44 & 22 & $\mathrm{rt}$ & $\mathrm{V}_{2}+\mathrm{V}_{3}$ & 1. RS; 2. MVD + rhiz & 18 & 54 & 100 \\
\hline 15 & $58, \mathrm{M}$ & 53 & 62 & $\mathrm{rt}$ & $\mathrm{V}_{2}^{2}$ & 1. MVD; $2 . \mathrm{RS}$ & 36 & 48 & 75 \\
\hline 16 & $63, \mathrm{M}$ & 58 & 59 & lt & $\mathrm{V}_{2}^{2}+\mathrm{V}_{3}$ & 1. MVD; 2. RF; 3. RF & 9 & 15 & 25 \\
\hline 17 & $56, \mathrm{~F}$ & 48 & 29 & lt & $\mathrm{V}_{1}^{2}+\mathrm{V}_{2}^{3}$ & 1. RS; 2. RF & 54 & 63 & 100 \\
\hline 18 & $57, \mathrm{M}$ & 46 & 65 & $\mathrm{rt}$ & $\mathrm{V}_{3}$ & 1. MVD; 2. RF & 2 & 67 & 75 \\
\hline 19 & $86, \mathrm{~F}$ & 77 & 24 & lt & $\mathrm{V}_{2}+\mathrm{V}_{3}$ & 1. RF; 2. MVD; 3. RF & 36 & 78 & 100 \\
\hline 20 & $71, \mathrm{M}$ & 59 & 74 & $\mathrm{rt}$ & $\mathrm{V}_{2}^{2}+\mathrm{V}_{3}$ & 1. MVD; 2. MVD + rhiz & 72 & 78 & 100 \\
\hline 21 & $74, \mathrm{M}$ & 65 & 111 & $\mathrm{rt}$ & $\mathrm{V}_{2}^{2}$ & 1. MVD; 2. MVD & 12 & 25 & 100 \\
\hline 22 & $76, \mathrm{~F}$ & 59 & 150 & $\mathrm{rt}$ & $\mathrm{V}_{3}^{2}$ & 1. RS; 2. MVD + rhiz & 18 & 54 & 25 \\
\hline 23 & $90, \mathrm{~F}$ & 78 & 98 & lt & $\mathrm{V}_{2}^{3}$ & 1. RS; $2 . \mathrm{RS}$ & 2 & 72 & 50 \\
\hline 24 & $57, \mathrm{~F}$ & 51 & 46 & lt & $\mathrm{V}_{2}^{2}$ & 1. MVD; $2 . \mathrm{RS}$ & 12 & 27 & 90 \\
\hline 25 & $83, \mathrm{M}$ & 47 & 444 & $\mathrm{rt}$ & $\mathrm{V}_{2}^{2}$ & 1. MVD + rhiz; 2. RS; 3. RS & 2 & 29 & 75 \\
\hline 26 & $57, \mathrm{M}$ & 41 & 146 & lt & $\mathrm{V}_{3}^{2}$ & 1. MVD + rhiz; 2. RF; 3. RS & 14 & 28 & 75 \\
\hline 27 & $57, \mathrm{M}$ & 37 & 243 & $\mathrm{rt}$ & $\mathrm{V}_{2}$ & 1. MVD; $2 . \mathrm{RS}$ & 12 & 40 & 50 \\
\hline 28 & $68, \mathrm{M}$ & 51 & 204 & $\mathrm{rt}$ & $\mathrm{V}_{3}^{2}$ & 1. MVD; 2. RS & 24 & 38 & 100 \\
\hline 29 & $67, \mathrm{~F}$ & 47 & 197 & $\mathrm{rt}$ & $\mathrm{V}_{3}$ & 1. RF; 2. MVD; 3. RS & 24 & 52 & 100 \\
\hline 30 & $72, \mathrm{M}$ & 65 & 48 & $\mathrm{rt}$ & $\mathrm{V}_{2}$ & 1. RS; 2. MVD & 36 & 43 & 50 \\
\hline 31 & $78, \mathrm{~F}$ & 60 & 189 & lt & $\mathrm{V}_{3}^{2}$ & 1. MVD + rhiz; 2. RS; 3. RS & 36 & 72 & 75 \\
\hline 32 & $51, \mathrm{~F}$ & 30 & 231 & lt & $\mathrm{V}_{2}$ & 1. MVD; 2. RF & 6 & 37 & 100 \\
\hline
\end{tabular}

$* \mathrm{FU}=$ follow up; $\mathrm{RF}$ = radiofrequency ablation; rhiz = partial rhizotomy; $\mathrm{RS}=$ radiosurgery; $\mathrm{Sx}=$ symptom; $\mathrm{Tx}=$ treatment. 
lar for retreated patients and for those who did not undergo additional procedures: 104.1 and 93.9 months, respectively $(p=0.278)$. In our sample there was no significant difference between the retreated group and those who did not undergo retreatment with regard to age $(p=0.347)$, duration of preoperative symptoms $(\mathrm{p}=0.278)$, or pain on the left side of the face $(\mathrm{p}=0.370)$ in men. Hence, outcomes did not differ significantly in the patients who underwent retreatment and those who did not, although the duration of preoperative symptoms was longer for patients in the retreated group.

Approximately $75 \%$ of the patients described their pain as sharp or jabbing, and half described it as similar to an electric shock (Table 2). To a lesser extent, patients described their pain as burning, aching, or dull. Preoperative numbness in the trigeminal distribution was present in $12.5 \%$ of patients. The number of patients with pain in the $\mathrm{V}_{2}$ or $\mathrm{V}_{3}$ distributions was the same (18 each), and five had pain in the $\mathrm{V}_{1}$ distribution; some patients had pain in more than one distribution.

The initial treatment for the 209 patients in our database was MVD with or without rhizotomy in 93 patients, radiofrequency ablation in 12, and radiosurgery in 104 (Table 3). The mean number of retreatments was $1.80 \pm 0.92$ per patient. The mean time between the initial treatment and the second one was $13 \pm 23$ months, and the mean time between the second and third treatment was $149 \pm 38$ months. No patient required more than two retreatments per affected side. The mean follow-up period between the first and last treatments was comparable for radiosurgery and radiofrequency ablation, and it was longer for those two treatments than for MVD, but this was not statistically significant.

Nineteen $(20.4 \%)$ of 93 patients who had initially undergone MVD required retreatment; 11 of these patients required one retreatment and eight required a second and final procedure (Tables 1 and 4). The final treatment in this group was radiosurgery for 14 patients, radiofrequency ablation for three, and MVD for two. Five (41.6\%) of 12 patients who initially underwent treatment with radiofrequency ablation were retreated; two of those patients required two retreatments. The final treatment for

TABLE 2

Demographic data in

32 patients who underwent retreatment for TN

\begin{tabular}{lc}
\hline \hline \multicolumn{1}{c}{ Characteristic } & Value (\%) \\
\hline male & $13(40.6)$ \\
female & $19(59.4)$ \\
mean age (yrs) & $69.8 \pm 12.3$ \\
mean age at onset (yrs) & $53.7 \pm 15.5$ \\
common symptoms & \\
sharp/jabbing pain & $23(71.9)$ \\
electrical shock-like pain & $16(50.0)$ \\
burning & $6(18.8)$ \\
numbness & $4(12.5)$ \\
aching & $4(12.5)$ \\
dull pain & $1(3.1)$ \\
distribution & $5(15.6)$ \\
$\mathrm{V}_{1}$ & $18(56.3)$ \\
$\mathrm{V}_{2}$ & $18(56.3)$ \\
$\mathrm{V}_{3}$ & \\
\hline
\end{tabular}

TABLE 3

Retreatment modalities and follow-up duration in 32 patients with TN

\begin{tabular}{lcccc}
\hline \hline & & & \multicolumn{2}{c}{ Mean FU in Mos (range) } \\
\cline { 3 - 5 } \multicolumn{1}{c}{ Tx } & $\begin{array}{c}\text { Total No. } \\
\text { Treated }\end{array}$ & Retreated (\%) & \multicolumn{1}{c}{ 1st Tx } & Last Tx \\
\hline MVD & 93 & $19(20.4)$ & $43 \pm 18(12-78)$ & $17 \pm 17(2-72)$ \\
RF & 12 & $5(41.6)$ & $60 \pm 16(49-78)$ & $30 \pm 10(18-36)$ \\
RS & 104 & $8(7.7)$ & $60 \pm 34(10-132)$ & $28 \pm 21(2-72)$ \\
\hline
\end{tabular}

this group was radiosurgery for four patients and repeated radiofrequency ablation for one. Eight (7.7\%) of 104 patients who were initially treated with radiosurgery required retreatment; seven required one procedure and two required a second retreatment. The final treatment for these patients was MVD for four, radiofrequency ablation for two, and radiosurgery for two. The patient in Case 5 had bilateral TN and required two treatments with radiosurgery and one treatment with MVD on each side (Table 1). The mean initial radiation dose given in radiosurgery was $77 \mathrm{~Gy}$, and the mean retreatment radiation dose was $40 \mathrm{~Gy}$.

Radiosurgery was the final treatment in 20 of 32 patients; $14(73.7 \%)$ of them reported 75 to $100 \%$ pain relief and five had 50\% relief (Tables 1 and 4). Microvascular decompression was the final treatment for six patients; four $(66.7 \%)$ attained $100 \%$ pain relief and two had 25 to $50 \%$ relief (Tables 1 and 5). Radiofrequency ablation was the final treatment in six patients; five of them (83\%) had 75 to $100 \%$ pain relief. To assess the efficacy of each modality as the final treatment, we calculated the percentage of times that a given retreatment was the final treatment for a given patient. We found that MVD was the final treatment in seven $(63.6 \%)$ of 11 , radiofrequency ablation in six $(66.7 \%)$ of nine, and radiosurgery in 19 (76\%) of 25 instances. (These numbers mean that when a given modality was used as a retreatment [that is, not the initial treatment] it was the last treatment $\mathrm{x} / \mathrm{y}[\%]$ of the time.)

After the final treatment, eight patients attained complete pain relief; three of them tapered off all medications, and five reduced their preoperative medications (Table 5). The other 24 experienced a mean of $85 \pm 12 \%$ pain relief, and of those, 13 were able to reduce their medications. Only one patient required increased amounts of medication. Of the eight patients who experienced complete pain relief, all reported worsening or new facial numbness. Two of the 23 patients who experienced improvement but not complete relief also had worsening or new facial numbness. There were no surgical complications in any of the patients who underwent retreatment.

TABLE 4

Number and type of retreatments in 32 patients with TN

\begin{tabular}{lccccccr}
\hline \hline & & \multicolumn{3}{c}{$\begin{array}{c}\text { No. of } \\
\text { Retreatments }\end{array}$} & & \multicolumn{3}{c}{ Final Tx } \\
\cline { 3 - 4 } \cline { 6 - 8 } Tx & No. of Patients & 1 & 2 & & MVD & RF & RS \\
\hline RF & 5 & 3 & 2 & & 0 & 1 & 4 \\
RS & 9 & 7 & 2 & & 5 & 2 & 2 \\
MVD & 18 & 10 & 8 & & 2 & 3 & 13 \\
\hline
\end{tabular}


R. O. Sanchez-Mejia, et al.

TABLE 5

Postoperative pain and medication use after last procedure in 32 patients who underwent retreatment for TN

\begin{tabular}{|c|c|c|c|c|c|c|c|c|}
\hline \multirow[b]{2}{*}{ Pain Level } & \multicolumn{4}{|c|}{ Medications } & \multirow{2}{*}{$\begin{array}{c}\text { Mean } \\
\text { \% Pain Relief }\end{array}$} & \multicolumn{2}{|c|}{ Mean FU (mos) } & \multirow{2}{*}{$\begin{array}{c}\text { Worsened } \\
\text { Postop Paresthesias }\end{array}$} \\
\hline & None & Reduced & Same & Increased & & From 1st Tx & From Last Tx & \\
\hline pain free & 3 & 5 & 0 & 0 & 100 & 33 & 16 & 8 \\
\hline reduced pain & 0 & 13 & 10 & 1 & 85 & 47 & 10 & 2 \\
\hline
\end{tabular}

Kaplan-Meier curves comparing rates of retreatment for each initial surgical procedure (MVD with or without partial rhizotomy, radiofrequency ablation, or radiosurgery) were plotted over time, beginning with the initial treatment (Fig. 1). Patients were categorized according to the first surgery they received. Radiosurgery required the fewest second treatments overall, and most patients experienced refractory pain by 40 months or less. Next to radiosurgery, MVD required the smallest number of second treatments.

\section{DISCUSSION}

Trigeminal neuralgia that is refractory to medical management can be treated initially with one of several therapies. The selection of a particular treatment is based on a variety of variables, including patient age, medical condition, the surgeon's suspicion of vascular compression of the trigeminal nerve root, and patient preference.

The treatment of medically refractory TN is not always successful, and patients may require retreatment. ${ }^{2,12,14} \mathrm{Re}-$

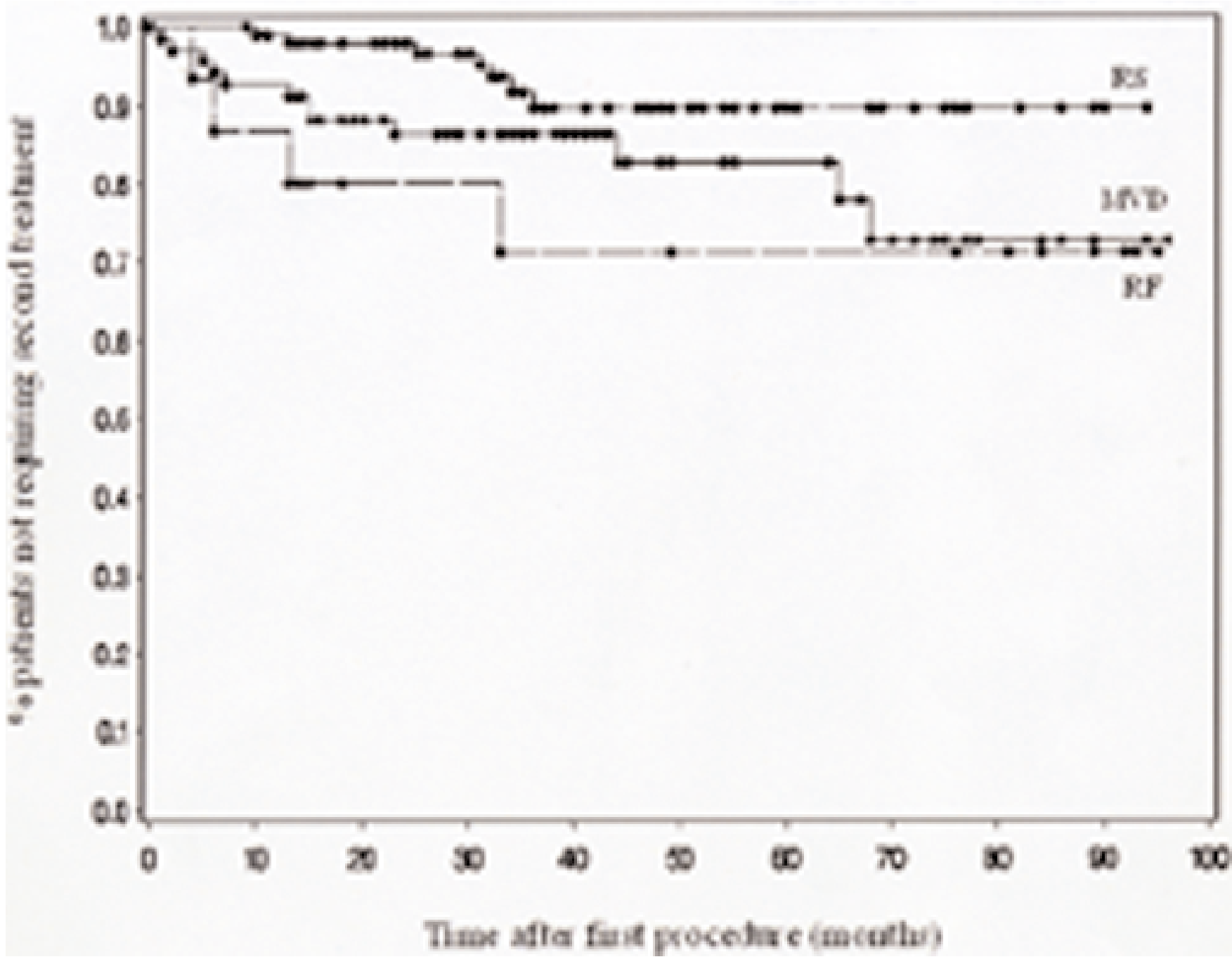

Fig. 1. Kaplan-Meier curves comparing the percentage of patients not requiring a second treatment with time elapsed after the first treatment. Patients receiving radiosurgery (RS) as the first treatment required the fewest second treatments. Patients undergoing MVD initially required fewer second treatments, but after 70 months the outcome was indistinguishable from that for radiofrequency ablation (RF). 
current pain is reported in 10 to $30 \%$ of patients, and $90 \%$ of the time it occurs in the distribution in which the initial symptoms appeared..$^{14}$ Recurrent symptoms have been associated with younger patient age at the time of surgery, longer preoperative duration of symptoms, venous compression of the trigeminal root entry zone, female sex, and pain on the left side of the face in men. ${ }^{2,9,14}$ In our patient cohort, we also had more women than men, but we found no statistical significance for any of the other variables. It is not known why women have a higher pain recurrence rate than men.

The lowest rate of retreatment was seen after radiosurgery (the follow-up periods for all treatment modalities were comparable). Although follow-up duration for the MVD treatment group was shorter, it was still long enough to allow evaluation for recurrence or refractory symptoms, given that the mean time between the initial and second treatment was approximately 13 months. Nevertheless, we cannot exclude the possibility that the shorter follow-up time for MVD led us to underestimate recurrence and the need for retreatment. The retreatment rate $(20.4 \%)$ after MVD in our series is comparable to the 10 to $20 \%$ rate reported in the literature. ${ }^{1,2,4}$ Radiofrequency ablation was associated with the highest rate of retreatment $(41.6 \%)$. We did not treat many patients with this modality because we believe that it leads to a very high incidence of sensory hypalgesia compared with radiosurgery or MVD. Our rate of retreatment after radiofrequency ablation was higher than the $15 \%$ reported in the literature. ${ }^{13,15}$ We do not know whether this is due to differences in patient selection or the number of treatments performed.

We had the lowest rate of retreatment $(7.7 \%)$ after radiosurgery. In contrast, others have reported that approximately 13 to $22 \%$ of patients require repeated treatment for recurrent symptoms after undergoing radiosurgery., 3,716 Although randomized blinded studies with comparable patient selection criteria would be necessary to compare the three treatment modalities statistically, our data indicate that radiosurgery may be more efficacious than MVD or radiofrequency ablation as an initial treatment for idiopathic TN. Whether the degree of this benefit would differ after correcting for patient characteristics such as age, identification of vessel involvement by magnetic resonance imaging, or other factors remains to be determined. Clearly, preoperative variables contribute to selection of the treatment modality, and further multivariate analysis will be needed to resolve this possibility.

When patients require retreatment, all of the initial treatment modalities are available. When MVD, radiosurgery, and radiofrequency ablation were given as retreatments, there was a $63.6,76$, and $66.7 \%$ chance, respectively, that no further treatment would be necessary. Radiosurgery was the final treatment in the majority of patients (19 of 32). Repeated MVD for recurrent or persistent pain can provide excellent or good results in approximately 75 to $85 \%$ of patients. ${ }^{2,9}$ In $56 \%$ of patients undergoing repeated MVD, vascular compression is observed again. Of the 19 patients who required retreatment after initial MVD in our study, in $72.2 \%$ radiosurgery was their final treatment. Shetter, et al. ${ }^{10}$ report that after a second radiosurgery treatment in which the gamma knife was used, 53\% of patients were pain- and medication-free, $21 \%$ were free of pain but still used medications, and $11 \%$ had incomplete pain control.
All retreatment modalities used in our cohort resulted in a similar percentage of patients with 75 to $100 \%$ pain control. Nevertheless, even though radiosurgery yielded pain control rates that were similar to those found for other modalities, it had a greater likelihood of being the final treatment; in our cohort, it was the final treatment in the majority of patients.

Trigeminal numbness is known to increase after any neurosurgical treatment of TN. Radiofrequency ablation leads to the highest rates of trigeminal numbness, followed by radiosurgery. Shetter, et al., reported a $42 \%$ incidence of new trigeminal numbness after a second neurosurgical procedure. Interestingly, patients in their study who had facial numbness had a greater likelihood of being pain free. Hasegawa, et al., ${ }^{6}$ described their experience treating refractory $\mathrm{TN}$ with radiosurgery after it had been previously treated with various modalities. They identified patients who experienced pain relief after the second (64-Gy) but not the first (75-Gy) treatment. Not surprisingly, they noticed a higher incidence of paresthesias after the second procedure. Similarly, in our patient cohort, all participants who had reported complete pain relief had worse or new trigeminal numbness, and only two of 24 patients who did not have complete relief had worse or new trigeminal numbness. Hence, postoperative trigeminal numbness correlated with improved relief of TN.

After plotting the need for retreatment after the initial procedure against the time elapsed since the initial treatment, it appears that radiosurgery requires retreatment less often than other modalities (Fig. 1), whereas MVD and radiofrequency ablation seem to have the same long-term efficacy. These results cannot be interpreted at face value because we did not correct for patient variables and how they affect patient selection for each treatment modality. Nevertheless, the results reflect, to some extent, the efficacy of the initial treatment within our current process of triage to different treatment modalities. Evaluation of preoperative variables and how they may contribute to treatment outcome will likely clarify this. Of course, a prospective, randomized, double-blind controlled study would allow for a better comparison between treatments.

\section{CONCLUSIONS}

Trigeminal neuralgia can recur after neurosurgical treatment. Our study indicates that the number of patients requiring retreatment is significant. Lower retreatment rates were seen with patients who initially underwent radiosurgery compared with MVD or radiofrequency ablation. Radiosurgery was more likely to be the final treatment for recurrent TN regardless of the initial treatment. After retreatment, the majority of patients reported complete or very good relief of pain. Pain relief after retreatment correlates with postoperative facial numbness.

\section{References}

1. Barker FG, Jannetta PJ, Bissonette DJ, et al: The long-term outcome of microvascular decompression for trigeminal neuralgia. N Engl J Med 334:1077-1084, 1996

2. Bederson JB, Wilson CB: Evaluation of microvascular decompression and partial sensory rhizotomy in 252 cases of trigeminal neuralgia. J Neurosurg 71:359-367, 1989 
3. Brisman R: Repeat gamma knife radiosurgery for trigeminal neuralgia. Stereotact Funct Neurosurg 81:43-49, 2003

4. Burchiel KJ, Clarke H, Haglund M, et al: Long-term efficacy of microvascular decompression in trigeminal neuralgia. J Neurosurg 69:35-38, 1988

5. Goetz CG: Textbook of Clinical Neurology, ed 2. Philadelphia: WB Saunders, 2003

6. Hasegawa T, Kondziolka D, Spiro R, et al: Repeat radiosurgery for refractory trigeminal neuralgia. Neurosurgery 50:494-502, 2002

7. Herman JM, Petit JH, Amin P, et al: Repeat gamma knife radiosurgery for refractory or recurrent trigeminal neuralgia: treatment outcomes and quality-of-life assessment. Int J Radiat Oncol Biol Phys 59:112-116, 2004

8. Jannetta PJ, Levy EI: Trigeminal neuralgia: microvascular decompression of the trigeminal nerve for tic douloureux, in Winn HR (ed): Youman's Neurological Surgery, ed 5. Philadelphia: WB Saunders, 2004, Vol 3, pp 3005-3015

9. Rath SA, Klein HJ, Richter HP: Findings and long-term results of subsequent operations after failed microvascular decompression for trigeminal neuralgia. Neurosurgery 39:933-940, 1996

10. Shetter AG, Rogers CL, Ponce F, et al: Gamma knife radiosurgery for recurrent trigeminal neuralgia. J Neurosurg (Suppl 5) 97:536-538, 2002

11. Sweet WH: The history of the development of treatment for trigeminal neuralgia. Clin Neurosurg 32:294-318, 1985
12. Sweet WH: The treatment of trigeminal neuralgia (tic douloureux). N Engl J Med 315:174-177, 1986

13. Taha JM, Tew JM Jr, Buncher CR: A prospective 15-year follow up of 154 consecutive patients with trigeminal neuralgia treated by percutaneous stereotactic radiofrequency thermal rhizotomy. J Neurosurg 83:989-993, 1995

14. Theodosopoulos PV, Marco E, Applebury C, et al: Predictive model for pain recurrence after posterior fossa surgery for trigeminal neuralgia. Arch Neurol 59:1297-1302, 2002

15. Tronnier VM, Rasche D, Hamer J, et al: Treatment of idiopathic trigeminal neuralgia: comparison of long-term outcome after radiofrequency rhizotomy and microvascular decompression. Neurosurgery 48:1261-1268, 2001

16. Urgosik D, Liscak R, Novotny J Jr, et al: Treatment of essential trigeminal neuralgia with gamma knife surgery. J Neurosurg (Suppl 1) 102:29-33, 2005

Manuscript received March 23, 2005.

Accepted in final form April 15, 2005.

Address reprint requests to: Nicholas M. Barbaro, M.D., Department of Neurological Surgery, University of California, San Francisco, 505 Parnassus Avenue, San Francisco, California 941430112. email: barbaron@ @eurosurg.ucsf.edu. 